\title{
Vorwort des Herausgebers
}

\author{
Ralf Thomas Münnich ${ }^{1}$
}

(C) Springer-Verlag Berlin Heidelberg 2016

Am 21. November 2015 verstarb überraschend Prof. Dr. Jürgen Wolters. In seiner Forschung beschäftigte sich unser Kollege vor allem mit der Ökonometrie und der Anwendung ökonometrischer Methoden auf Fragestellungen aus der Geld- und Wirtschaftspolitik, so beispielsweise Untersuchungen zur Zinsstruktur sowie zum geldpolitischen Transmissionsprozess und zu Konjunkturschwankungen. Gerade in der aktuellen Zeit, die von den Notenbanken weltweit eine unkonventionelle Geldpolitik verlangt, zeigt sich die Wichtigkeit dieses Forschungszweigs. Seit 1982 und bis zu seiner Pensionierung 2008 war Jürgen Wolters Professor für Ökonometrie an der Freien Universität Berlin. Von 1999 bis 2003 war er Vorsitzender des Ausschusses für Empirische Wirtschaftsforschung und Angewandte Ökonometrie in der Deutschen Statistischen Gesellschaft. Auch als Emeritus blieb Jürgen Wolters der Forschung als Research Fellow am Schweizerischen Institut für Aussenwirtschaft und Angewandte Wirtschaftsforschung an der Universität St. Gallen treu. Ein Nachruf auf Jürgen Wolters wurde von seinem Schüler Uwe Hassler verfasst (Hassler 2016).

Im Vorfeld des Weltwirtschaftsforums in Davos im Januar 2016 wurde eine Studie der Entwicklungsorganisation Oxfam vorgestellt. Eine der Kernaussagen der Studie ist, dass in 2014 das reichste Prozent der Weltbevölkerung ca. $48 \%$ des weltweiten Gesamtvermögens besaß. Zudem soll sich das Vermögen der 80 reichsten Einzelpersonen von 2009 bis 2014 ungefähr verdoppelt haben. Ohne diese Studie genau zu durchleuchten, zeigt selbige doch die Brisanz des Themenkomplexes Ungleichheit. Eine präzise Erfassung des Phänomens setzt ein geeignetes Instrumentarium voraus. Kockläuner (2013) berichtete bereits 2013 im AStA - Wirtschafts- und Sozialstatistisches Archiv über den Einbezug von Ungleichheitsmaßen in den Bericht über die menschliche Entwicklung seit 2010. In Kockläuner (2016) zeigt er

Ralf Thomas Münnich

muennich@uni-trier.de

1 Wirtschafts- und Sozialstatistik, Fachbereich IV, VWL, Universität Trier, Trier, Deutschland 
nun im Überblick die gemeinsame Struktur vieler relativer Ungleichheitsmaße auf. Sie zeichnen sich dabei durch einen auf verschiedenen Lagemaßen oder höheren Momenten basierenden relativen Vergleich aus. Konkret betrachtet der Autor neben dem weithin bekannten Gini-Koeffizienten die Ungleichheitsmaße von Atkinson und Kolm sowie die informationstheoretisch begründeten Entropiemaße von Theil. Weitere Ungleichheitsmaße, die den gemeinsamen Rahmen der vorgenannten Maße sprengen, listet der Autor ebenso auf.

Der Anteil erneuerbarer Energien am bundesdeutschen Strommix soll laut Koalitionsvertrag der Großen Koalition bis zum Jahr 202540 bis $45 \%$ erreicht haben. Auch im gesamten europäischen Raum werden ähnliche Ziele verfolgt. Laut vorläufigen Angaben des Statistischen Bundesamtes lag der Anteil der erneuerbaren Energien am Bruttostromverbrauch in 2015 bereits bei 30\%; 2005 waren es noch gut $10 \%$. Den größten Einzelbeitrag im Bereich der Erneuerbaren liefert dabei die Windkraft mit ihren On- und Off-Shore-Anlagen. Zur gezielten Planung des Baus neuer Anlagen und zur Absicherung der Energieversorgung sind in diesem Umfeld auch präzise kurz- und mittelfristige Vorhersagen der Windgeschwindigkeit und der von dieser im Wesentlichen bestimmten Windenergie vonnöten. Mit Blick auf die staatlich garantierte Einspeisevergütung haben die Teilnehmer am Energiemarkt ebenfalls ein starkes Interesse an solchen Prognosen. In ihrem Beitrag für das AStA Wirtschafts- und Sozialstatistisches Archiv wenden Ambach und Garthoff (2016) ein periodisch-saisonales vektorautoregressives Prognosemodell (VAR) zur Vorhersage der mittleren Windgeschwindigkeit in einem bestimmten Windpark in Ostdeutschland an; der maximale Prognosehorizont beträgt dabei $48 \mathrm{~h}$. Eine Besonderheit ist dabei die Nutzung auf Schwellenwerten basierender autoregressiver bedingter Heteroskedastizität (TARCH). Die Schätzung der Modelle erfolgt mithilfe der LASSO-Methode mit iterativer Neugewichtung. Diese ermöglicht kürzere Berechnungszeiten als die Maximum-Likelihood-Methode. Ausgehend von den Geschwindigkeitsprognosen wird letztlich die Windenergie vorhergesagt. Ergänzt wird der Beitrag durch einen Vergleich der eingesetzten mit weiteren Schätzmethoden, sowohl innerhalb als auch außerhalb der Stichprobe.

Die Nutzer vertraulichen Datenmaterials und der Gesetzgeber haben in der Regel diametral entgegengesetzte Interessen. Der Datennutzer möchte idealerweise unmittelbar mit den Originaldaten arbeiten. Sofern dies nicht möglich ist, sollte für ihn der Informationsverlust im Zuge einer Veränderung der Daten durch den Datenhalter möglichst gering sein. Der Gesetzgeber, und natürlich auch die hinter den Daten stehenden Personen, Haushalte, Unternehmen etc., wollen andererseits anonym bleiben und das Reidentifikationsrisiko minimiert wissen, was für eine starke Verfremdung der Daten sprechen würde. In der Praxis gilt es somit, die beiden Interessen möglichst gut auszubalancieren. Dieser Themenkomplex gewinnt in Zeiten der Verfügbarkeit immer größerer Datenmengen an Beachtung und Wichtigkeit. Lenz (2016) beschäftigt sich in seinem Aufsatz für AStA Wirtschafts- und Sozialstatistisches Archiv mit dieser Problematik im Kontext von Fallzahltabellen. Dieses Thema wird von Forschern bereits seit den 1960er-Jahren beleuchtet. Hier werden traditionell sowohl prä- als auch posttabulare Geheimhaltungsstrategien angewandt. Die letztgenannten Strategien zeichnen sich dabei durch Modifikationen von auf Originaldaten erzeugten Tabellen aus. Dabei kommen verschiedene Methoden der Sperrung, Rundung und Überlagerung 
von Zellwerten zum Einsatz. Die Verfahrensgruppe der sogenannten zyklischen Überlagerung scheint hier besonders geeignet zu sein. Mithilfe von Zufallszahlen wird dabei eine Folge von Überlagerungsmustern sukzessive zur Ausgangstabelle addiert, wobei nicht nur Zellen mit kleiner Belegung verändert werden, sondern sämtliche Zellen. Ein Vorteil ist dabei die mögliche Bewahrung der Randhäufigkeiten. Die Konsistenz zwischen Tabellen kann dann allerdings nur noch approximativ erreicht werden, was die sprichwörtliche Kehrseite der Medaille darstellt. Auch hier muss demnach eine Wahl getroffen werden. Der Autor stellt in seinem Beitrag diverse Variationen der zyklischen Überlagerung vor und bewertet diese mit Blick auf den oben genannten Zielkonflikt. Weiterhin gibt Lenz Hinweise zur praktischen Anwendung der Technik.

Auch im Jahr 2016 geht die von Walter Krämer initiierte Interview-Serie weiter (Krämer 2016). In dieser Ausgabe des AStA Wirtschafts- und Sozialstatistisches Archiv finden Sie eine Unterhaltung von ihm mit dem zuletzt bis zu seiner Emeritierung 2014 an der Universität zu Köln in den Bereichen Statistik und Ökonometrie forschenden und lehrenden Karl Mosler. Seine Forschungsschwerpunkte setzte der Befragte in den Bereichen der nichtparametrischen Analyse multivariater Daten, der Modellierung von Risiko sowie der Messung sozio-ökonomischer Ungleichheit. Jenseits der Forschung und Lehre engagierte sich Herr Mosler für die deutsche Statistik in seinen Rollen als Präsident der Deutschen Statistischen Gesellschaft (2004-2008) sowie als Mitgründer und stellvertretender Vorsitzender der Deutschen Arbeitsgemeinschaft Statistik - DAGStat - (seit 2005). Von 1998 bis 2004 gab er die internationale Schwesterzeitschrift des AStA Wirtschafts- und Sozialstatistisches Archiv, das AStA: Advances in Statistical Analysis heraus. Mit Herrn Mosler traf sich Walter Krämer am Rande der Statistischen Woche 2014 in Hannover. Im Interview lesen Sie über Herrn Moslers Erinnerungen an die gemeinsamen Arbeiten mit Gleb Koshevoy und Rainer Dyckerhoff und über die Geburtsstunde der Zeitschrift die Sie gerade in den Händen halten. Weiterhin diskutiert er mit Walter Krämer über die zulässige Spannbreite der Bezeichnung Statistik, eine merkwürdige deutsche Eigenart und die Zukunft der Statistikausbildung an deutschen Universitäten.

Und nun wünsche ich Ihnen, liebe Leserinnen und Leser, viel Spaß bei der Lektüre der ersten Ausgabe von AStA Wirtschafts- und Sozialstatistisches Archiv im Jahr 2016.

\section{Literatur}

Ambach D, Garthoff R (2016) Vorhersagen der Windgeschwindigkeit und Windenergie in Deutschland. AStA Wirtsch Sozialstat Arch 10(1). doi:10.1007/s11943-016-0177-1

Hassler U (2016) Nachruf auf Jürgen Wolters. AStA Wirtsch Sozialstat Arch 10(1). doi:10.1007/ s11943-016-0181-5

Kockläuner G (2013) Die neuen Indizes des Bericht über die menschliche Entwicklung 2010. AStA Wirtsch Sozialstat Arch 7(3-4):121-133

Kockläuner G (2016) Die gemeinsamen Strukturen relativer Ungleichheitsmaße. AStA Wirtsch Sozialstat Arch 10(1). doi:10.1007/s11943-016-0178-0

Krämer W (2016) Interview mit Karl Mosler. AStA Wirtsch Sozialstat Arch 10(1). doi:10.1007/ s11943-016-0179-z

Lenz R (2016) Recent advances in cyclic perturbation of frequency tables. AStA Wirtsch Sozialstat Arch 10(1). doi:10.1007/s11943-016-0180-6 